\title{
Advanced Information Technologies under Manifold Coordinate Systems
}

\author{
Volodymyr Riznyk \\ Automated Control Systems Department \\ Lviv Polynechnic National University \\ Lviv, Ukraine
}

Received: February 1, 2021. Revised: March 23, 2021. Accepted: March 30, 2021. Published: April 5, 2021.

\begin{abstract}
In this paper, we regard information technologies under manifold coordinate systems, namely the concept of Ideal Ring Bundles (IRBs), which can be used for configure of the system with the minimized basis for vector data coding and processing designs. The concept involves establishing harmonious mutual penetration of symmetry and asymmetry law as the remarkable property of real space, which allows optimize information technologies based on the law for finding optimal solutions for wide classes of technological problems in informatics, using novel designs based on combinatorial configurations such as the IRBs and their manifold topological transformations. These design techniques make it possible to configure big vector data information systems with fewer numbers of code words than at present, while maintaining or improving on transformation content and the other operating characteristics of the system by means of combinatorial optimization.
\end{abstract}

Keywords - combinatorial configuration, symmetry and asymmetry elegant ensemble, Ideal Ring Bundle, optimum vector data coding design, torus coordinate system, optimal vector code.

\section{INTRODUCTION}

Modern combinatorial design techniques and modeling are well using for finding optimal solution of wide classes of technological problems. However, the design based on the traditional combinatorial theory is not always applicable for information systems. In general case it was possible to take in consideration a new conceptual model of the systems, based on the idea of "perfect" combinatorial constructions. The problem, is known, to be of very important for configure information systems with fewer structural elements and bonds than at present, while maintaining or improving on resolving ability and the other operating characteristics of the system. The basic laws of worldwide harmony, such as Golden ratio [1] and Ideal Ring Bundles (IRBs) [2,3] to be of very profitable for development of fundamental and applied researches in vector signal processing [4], systems engineering [5], and manufacturing [6]. The developed concept associates with Ideal Ring Bundles, which is that a unity can be partitioned "perfectly" in ring sequence form, and the sums of connected sub-sequences of a unity enumerate the set of integers exactly $R$-times. This property makes IRBs extremely effective, when applied to the problem of finding the optimum ordered events in spatially or temporally distributed systems. Applications profiting from (IRBs) are for example one- and multidimensional vector data coding design, compression, and signal processing, and reconstruction [7]. The idea of partition sets with the smallest possible number of intersections is in agreement with describing the physics of toroidal confined plasmas [8]. In one dimension, a usual single-holed torus is the 1-torus [9] as a ring shape object. In two dimensions, we see a usual torus, also called the 2-torus. In analogy with this concept, in three and more dimensions, the $t$-dimensional torus, or $t$ manifold [10], is an object that exists in dimension $t+1$. This notion used to help visualize aspects of higher dimensional toroidal spaces. It was the torus mathematical model useful for describe geometric objects in spatial coordinates. The torus topology is superior to geometry for describing such objects because relate with philosophical spatial relationships. Many scientists also have suggested that the entire universe is a torus. Modern geometry is the study of spatial structures using Galois algebra [11], projective geometry [12], and combinatorial theory [13]. Concept of Ideal Ring Bundles involves techniques for developing the optimum vector data coding design for advanced computer systems and information technologies with respect to dimensionality, using novel designs based on cyclic groups of Galois field [11], projective geometry [11-12], and Ideal Ring Bundles [14].

\section{SYMmETRY AND ASYMMETRY ELEGANT ENSEMBLES}

\section{A. Symmetry and Asymmetry in Modern Sciences}

Symmetry and asymmetry relation in geometric structure is the most familiar type of them. The more general meaning of symmetry-asymmetry is in combinatorial configurations as a whole. In this context, symmetries and asymmetries underlie some of the most profound results found in modern physics, including aspects of space and time [1]. Finally, discusses interpenetrating symmetry and asymmetry in the humanities, covering its rich and varied use in architecture, philosophy, and art. Space-time symmetries are features of space-time that described as exhibiting some form of symmetry [15]. The role of symmetry in physics is important in simplifying solutions to many problems, e.g. exact solutions of Einstein's field equations of general relativity [16], and study of isometrics in two or three-dimensional Euclidian space [17]. 


\section{B. Elegant Symmetry and Asymmetry Ensembles}

Let us regard a sketch of $S$-fold rotational symmetry joined on two complementary asymmetries of the symmetry, where we require all angular distances between of straight lines emanated from a common point in each of the complementary asymmetries enumerate a set of two subsets of enumerated angles fixed number of times for each of the subsets.

A sketch of rotational symmetry of order seven $(S=7)$ joined on two complementary asymmetries and symmetry represented by three heavy and four thin straight lines given in Fig.1.

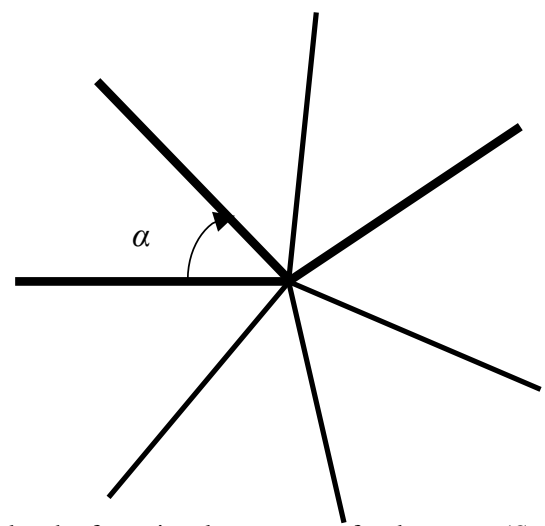

Fig. 1 A sketch of rotational symmetry of order seven $(S=7)$ joined on two complementary asymmetries of the symmetry represented by three thick and four thin straight lines.

The $S$ - fold rotational symmetry $(S=7)$ creates a spatial composition with three $\left(n_{1}=3\right)$ thick and four $\left(n_{2}=4\right)$ thin straight bunch lines, each of them contains asymmetrically flared beams in planar projection. Moving clockwise by spacing units $\alpha=360^{\circ} / S$ in arrow (Fig.1), we derive the next cyclic spacing integer sequences:

1) $\{1,1,1,1,1,1,1\}$ among seven $(S=7)$ single spacing units;

2) $\{1,2,4\}$ of three $\left(n_{1}=3\right)$ cyclic spacing among thick lines;

3) $\{2,1,1,3\}$ of four $\left(n_{2}=4\right)$ cyclic spacing among thin ones.

The cyclic sequence $\{1,2,4\}$ containing three $\left(n_{1}=3\right)$ elements allows an enumeration of all numbers $\mathbf{1}=1, \mathbf{2}=2$, $\mathbf{3}=1+2, \mathbf{4}, \mathbf{5}=4+1, \mathbf{6}=2+4, \mathbf{7}=1+2+4$ exactly once $\left(R_{1}=1\right)$, while $\{2,1,1,3\}$ containing four $\left(n_{2}=4\right)$ elements lets an enumeration the set of all integers $[1,6]$ exactly twice $\left(R_{2}=2\right): \mathbf{1}=1$ and $\mathbf{1}=1$, $\mathbf{2}=2$ and $\mathbf{2}=1+1, \mathbf{3}=3$ and $\mathbf{3}=2+1, \mathbf{4}=1+3$ and $\mathbf{4}=2+1+1, \mathbf{5}=3+2$ and $\mathbf{5}=1+1+3, \mathbf{6}=1+3+2$ and $\mathbf{6}=3+2+1$.

To see this, we observe the 7-fold rotational symmetry contains an informative parameters of two complementary Ideal Ring Bundles: 1) IRB $\{1,2,4\}$ with $S=7, n=3, R=1 ; 2) \operatorname{IRB}\{2,1$, 1,3 \} with $S=7, n=4, R=2$.

Definition. A set of angular spaces between everything possible pairs of $n$ lines diverged from common center of $S$-fold rotational symmetry, which allows an enumeration of integers $[1, S-1]$ exactly $R$-times, is the Elegant Symmetry and Asymmetry Ensemble (ESAE) [14].

The concept of Ideal Ring Bundles can be used for finding optimal solutions for wide classes of scientific and technological problems, including development of algebraic techniques, based on the idea of two- and multidimensional "perfect" combinatorial configurations.

We can notice the fundamental role of the real space as storage medium information about grandiose harmony of quantum structure of the world, and expect a better understanding of the role of topological combinatorial structures in the behavior of natural and artificial objects.

\section{INTELLIGENT TORUS COORDINATE SYSTEMS}

\section{A. Graph of Intelligent Torus Coordinate System}

Many researchers put forward that the Universe is a torus [9]. In case two $(t=2)$ dimensions, we recognize a natural torus. Let us regard a single-holed torus in 2-D space as a model of a planar ring shape system of $S$-fold rotational symmetry. Such model is the 1 - torus as a ring shape object of order $S$, namely an IRB with parameters $S, n, R$, where [14]:

$$
(S-1) R=n(n-1)
$$

Here is an example of the IRB $\{1,2,6,4\}$ containing four $(n=4)$ elements, which allows an enumeration of all numbers from 1 to $S-1=n(n-1)=12$ exactly once: $1,2,3=1+2,4$, $5=4+1, \ldots 12=2+6+4=13$ (Fig. 2 ).

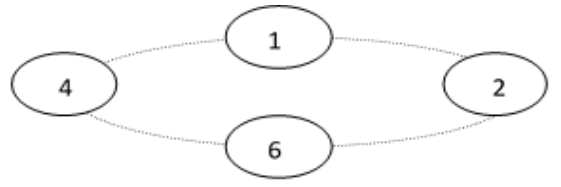

Fig. 2 Graph representation of the IRB $\{1,2,6,4\}$

From IRB $\{1,2,6,4\}$ follows intelligent cyclic relationship $1: 2: 6: 4$, which provides conceptual model of systems with nonuniform structure (e.g. binary cyclic weighted code), based on the useful combinatorial properties of planar state space partitions (Fig.3).

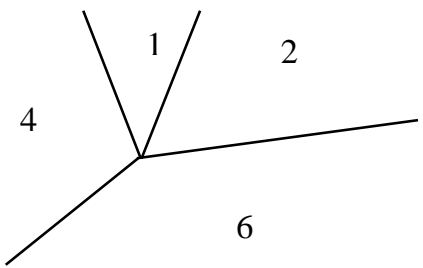

Fig.3 Graph representation of intelligent cyclic relationship 1:2:6:4.

Graphic interpretation of the intelligent cyclic relationship which property makes it possible to partition space with the smallest possible number of intersections.

\section{B. Intelligent Torus Coordinate System}

The key goal of the contemporary information systems is development of optimal systems with respect to improving their functionality using innovative conceptual models of higher dimensionality [14]. Additional aim of the system is formation of potential source for the development of higher organization levels in vector data processing and computing. In this aspect, the "perfect" torus coordinate system is of very useful.

Two-dimensional torus coordinate system formed by cyclic sequence $C$ of $n$ two-stage $(t=2)$ sub-sequences $\left\{K_{\mathrm{i}}\right\}=\left\{k_{\mathrm{i} 1}\right.$, 
$\left.k_{\mathrm{i} 2}\right\}, i=1,2, \ldots, n$, of the sequence $C=\left\{K_{1}, K_{2}, \ldots, K_{\mathrm{i}}, \ldots, K_{\mathrm{n}}\right\}$ (Fig.4).

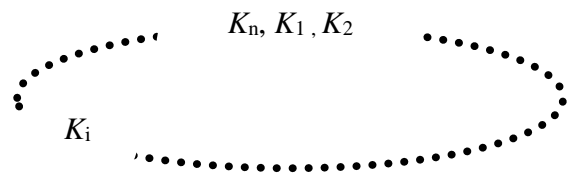

Fig.4 The two-dimensional cyclic $n$-stage sequence $C=\left\{K_{1}, K_{2}, \ldots, K_{\mathrm{i}}, \ldots\right.$, $\left.K_{\mathrm{n}}\right\}$ of two-stage sub-sequences $\left\{K_{\mathrm{i}}\right\}=\left\{k_{\mathrm{i} 1}, k_{\mathrm{i} 2}\right\}$ of the sequence, $(i=1$, $2, \ldots, n)$

Example: The cyclic sequence of three $(n=3)$ two-stage subsequences $K_{1}=(0,1), \quad K_{2}=(1,0), \quad K_{3}=(0,2)$ form a twodimensional $(t=2)$ "perfect" toroidal reference grid $m_{1} \times m_{2}=2 \times 3$ taking the mod 2 , and mod 3 sums of these two-stage sequences based on the combinatorial configuration $\{(0,1),(1,0),(0,2)\}$, namely the "Glory to Ukraine Star" (GUS), or starconfiguration [18] (Table 1).

TABLE I. INTELLIGENT TORUS COORDINATE GRID BASED ON THE CONFIGURATION $\{(0,1),(1,0),(0,2)\}$

\begin{tabular}{|c|c|c|c|c|}
\hline \multirow{2}{*}{$\mathbf{n} / \mathbf{n}$} & \multicolumn{5}{|c|}{ Forming the reference grid $m_{1} \times m_{2}=\mathbf{2} \times \mathbf{3}$} \\
\cline { 2 - 5 } & \multirow{2}{*}{ References } & \multicolumn{2}{|l|}{$\begin{array}{l}\text { Summation mod3, and mod2 of the 2-stage } \\
\text { sequences }\end{array}$} \\
\cline { 3 - 5 } & & $(\mathbf{0 , 1 )}$ & $(1,0)$ & $(\mathbf{0 , 2})$ \\
\hline $\mathbf{1}$ & $(0,0)$ & + & & + \\
\hline $\mathbf{2}$ & $(0,1)$ & + & & + \\
\hline $\mathbf{3}$ & $(0,2)$ & & & \\
\hline $\mathbf{4}$ & $(1,0)$ & & + & + \\
\hline $\mathbf{5}$ & $(1,1)$ & + & + & + \\
\hline $\mathbf{6}$ & $(1,2)$ & & + & + \\
\hline
\end{tabular}

From Table 1, easy to see the $2 \mathrm{D}$ cyclic sequence $\{(0,1),(1,0),(0,2)\}$ forms $2 \mathrm{D}$ torus reference grid $2 \times 3$, and a set $S_{1}$ of nodal dots of the two-dimensional coordinate grid one-toone corresponds to a set $S_{2}$ of $2 \mathrm{D}$ vector data attributes, the first numerical index indicates vector data by two $\left(m_{1}=2\right)$ categories, while the second - three $\left(m_{2}=3\right)$ of ones by the same vector.

In the case of two- dimensional $(t=2)$ vector data system it is a coordinate grid of fixed sizes $m_{1} \times m_{2}$ covered surface of usual torus with $(0,0)$ common point (Fig.5).

$m_{2}$

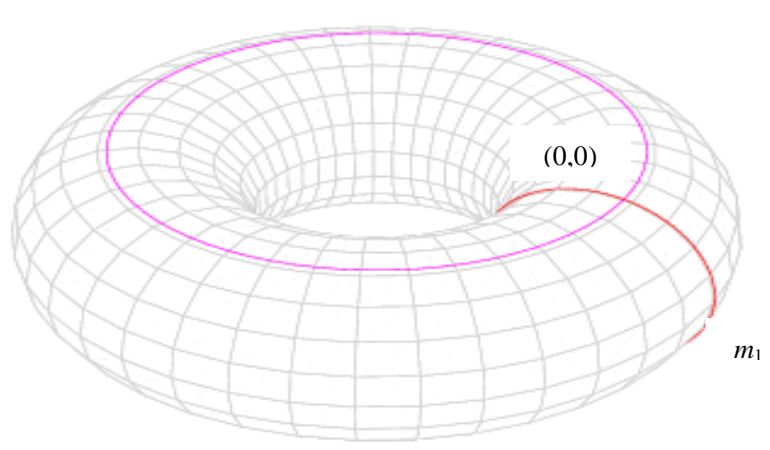

Fig.5 Torus coordinate grid $m_{1} \times m_{2}$ with $(0,0)$ common point

For example, binary $2 \mathrm{D}$ vector star-code based on the $\{(0,1),(0,2),(1,0)\}$ under torus grid $2 \times 3$ shows in Table 2 .

TABLE II. BinARY 2D Vector Code BASED on the $\{(0,1),(1,0),(0,2)\}$

\begin{tabular}{|c|c|c|c|c|}
\hline \multirow{2}{*}{$\mathbf{n} / \mathbf{n}$} & \multicolumn{5}{|c|}{ The star code under intelligent torus coordinate } \\
system $\boldsymbol{m}_{\mathbf{1}} \times \mathbf{m}_{\mathbf{2}}=\mathbf{2} \times \mathbf{3}$ \\
\cline { 2 - 5 } & $\begin{array}{c}\text { Code } \\
\text { combinations }\end{array}$ & \multicolumn{3}{|c|}{ Digit weights of the 2 D star code } \\
\cline { 3 - 5 } & $(0,0)$ & 1 & 0 & $(\mathbf{0 , 2})$ \\
\hline $\mathbf{1}$ & $(0,0)$ & 0 & 0 \\
\hline $\mathbf{2}$ & $(0,1)$ & 1 & 0 & 1 \\
\hline $\mathbf{3}$ & $(0,2)$ & 0 & 1 & 0 \\
\hline $\mathbf{4}$ & $(1,0)$ & 0 & 1 & 0 \\
\hline $\mathbf{5}$ & $(1,1)$ & 1 & 1 & 1 \\
\hline $\mathbf{6}$ & $(1,2)$ & 0 & & \\
\hline
\end{tabular}

Since star-configuration $\{(0,1),(0,2),(1,0)\}$ is multiplicative group of a finite field [11] it is complete set of binary $2 \mathrm{D}$ vector "Star" coding systems in four variants: $\{(1,0),(1,1),(1,2)\}$, $\{(0,1),(0,2),(1,0)\},\{(0,1),(0,2),(1,2)\},\{(0,1),(0,2),(1,1)\}$.

The underlying property of star-vector code allows on $2 \mathrm{D}$ vector data processing over the $2 \times 3$ range concurrently.

\section{INTELLIGENT MANIFOLD COORDINATE SYSTEMS}

\section{A. Model of manifold coordinate system}

Graphic representation of manifold reference grid $m_{1} \times m_{2}$ $\times \ldots \times m_{\mathrm{t}}$ formed by $t$-dimensional star-configuration about concurrent axes $m_{1}, m_{2}, \ldots, m_{\mathrm{t}}$ with common point "+" given in Fig.6.

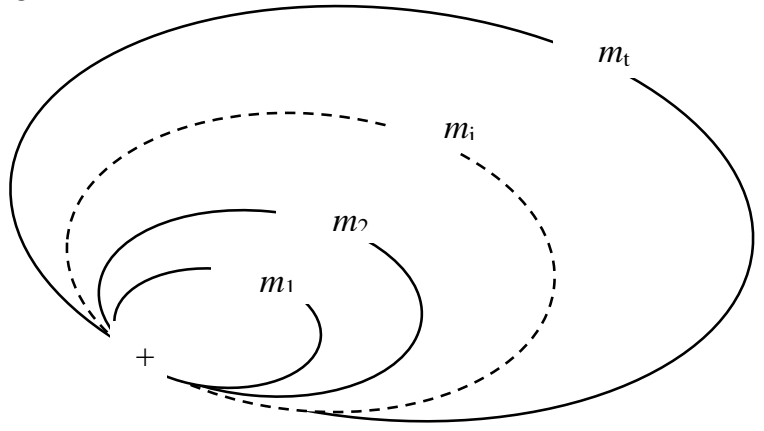

Fig.6 A planar projection of manifold grid $m_{1} \times m_{2} \times \ldots \times m_{\mathrm{t}}$ formed by $t$ dimensional star-configuration about concurrent axes $m_{1}, m_{2}, \ldots, m_{\mathrm{t}}$ with common point " + "

The "Star" codes may be separating as monolithic and group vector codes, and non-redundant vector codes [14]. First of them belong to the self-correcting codes due to monolithic arranged both symbols " 1 " and of course " 0 " for each allowed code word. This characteristic property offers at once detect or correct code words by the monolithic manner. The second "Star" codes are non-redundant ones, which provide processing and visualization of high-dimensional datasets.

There are a priory infinite set of "Star" codes under toroidal coordinate systems. The rate of number growth these codes 
with rising their size we can define tentatively by indexes of 2D and 3D self-correcting "Star" codes for $n=2, \ldots, 7$ from Table 3.

TABLE III. INDEXES OF 2D AND 3D STAR CODES

\begin{tabular}{|l|l|l|l|l|}
\hline \multirow{2}{*}{$\begin{array}{l}\text { Code } \\
\text { length, } \boldsymbol{n}\end{array}$} & \multicolumn{2}{|l|}{ Number of code variants } & \multicolumn{2}{l|}{ Code size variants } \\
\cline { 2 - 5 } & 2D codes & 3D codes & 2D codes & 3D codes \\
\hline 2 & 1 & - & $1 \times 2$ & - \\
\hline 3 & 4 & - & $2 \times 3$ & - \\
\hline 4 & 24 & - & $3 \times 4$ & - \\
\hline 5 & 272 & - & $4 \times 5,3 \times 7$ & - \\
\hline 6 & 256 & 128 & $5 \times 6,3 \times 10$ & $2 \times 3 \times 5$ \\
\hline 7 & 360 & 180 & $6 \times 7,3 \times 14$ & $2 \times 3 \times 6$ \\
\hline
\end{tabular}

Here we see fast growing varieties of "Star" codes under toroidal reference systems with rising their length $n$ and maximum code size $N_{\max }$

$$
N_{\max }=\prod_{1}^{t} m_{i}<2^{n},\left(m_{1}, m_{2}, \ldots, m_{\mathrm{t}}\right)=1
$$

The constraint (2) provides varies of "Star" codes under toroidal reference systems over a wide range, and graphic pictures of these systems offer a better understanding of the role of torus and manifold shape in the behaviour of natural and man-made objects. Remarkable combinatorial properties and structural perfection of star-configurations provide many opportunities to apply them to numerous branches of science and advance technology. Examples: vector computing and big data information technologies, code security communications, and vector data coding design $[4,14,18]$. The underlying skills is useful at high schools and universities for in-depth training of students, which study computer sciences and information technologies, involving contemporary combinatorial and algebraic theory for increasing interest to scientific researches.

\section{B. Optimal vector codes based on IRBs}

From the underlying equations (1) and (2) have been defined the next types of the optimum weighed $n$ - digital binary ring monolithic codes based on IRBs [18]:

a) numerical code is a set of ring $n$ - sequences of positive integers which sums of connected sub-sequences of the set enumerate the set of integers $[1, N]$ exactly $R$ - times, $N=n(n$ 1)/R

b) two-dimensional code is a set of ring $n$ - sequences of 2tuple nonnegative integers which modular sums of connected 2 - tuple taken modulo $m_{1}$ and $m_{2}$, respectively, allows an enumeration of nodal points of coordinate grid $m_{1} \times m_{2}$ over 3torus exactly $R$ - times with frame of axes $m_{1} \cdot m_{2}=n(n-1) / R$; c) multidimensional code is a set of ring $n$ - sequences of $t$-tuple nonnegative integers which modular sums of connected $t$ - tuple taken modulo $m_{1}, m_{2}, \ldots, m_{t}$, respectively, allows an enumeration of nodal points of coordinate grid $m_{1} \times \ldots \times m_{t}$ over $(t+1)$ - torus exactly $R$-times with frame of axes $m_{1} \cdot m_{2}$ $\ldots \cdot m_{t}=n(n-1) / R$.

Hence, $t$-dimensional star-configuration with $t$-dimensional torus or manifold coordinate grid $m_{1} \times m_{2} \times \ldots \times m_{\mathrm{t}}$ forms $t$ - dimensional vector "Star" codes, which code size equal to number of reference grid points.

The existence of an a priori of an infinitely large number of minimized basis, which give rise to numerous varieties of multidimensional star-code coordinate systems, opens up new possibilities for solving a wide range of mathematical and applied problems in contemporary informatics and computer science, cybernetics and management on the platform of system mathematics. We take into account the developments of modern theory of systems as a set of philosophical, methodological and scientific, and applied problems of analysis and synthesis of high advantageous vector information technologies.

\section{ACKNOWLEDGMENT}

This work involves the results performed in 2012-2017 years in Automated Control Systems Department of Lviv Polytechnic National University. I grateful to my colleagues for their active participation in the research presented in completed work on the R\&S project "Designing Software for Vector Data Processing and Information Protection Based on Combinatorial Optimization".

\section{REFERENCES}

[1] H. Weyl, Symmetry. Princeton, New Jersey: Princeton University Press, 1952

[2] V. Riznyk,"Perfect Distribution Phenomenon in Combinatorics and Systems Theory," in Proceedings of the 7-th Zittau Fuzzy Colloquium, September 8-10, Zittau, Germany, 1999, pp. 194-197.

[3] V.V. Riznyk, "Perfect Distribution Phenomenon and the Origin of the Spacetime Harmony," in International Conference on Generative Art, No 10825, Milano, Italy, 2001, http://papers.cumincad.org/cgibin/works/Show?ga0131

[4] S. Golomb, P. Osmera, and V. Riznyk, "Combinatorial Sequencing Theory for Optimization of Signal Data Vector Converting and Signal Processing," in Workshop on Design Methodologies for Signal Processing, Zakopane, 1996, pp. 43-44.

[5] V.V. Riznyk, and S.W. Golomb, "Researches and Applications of the Combinatorial Configurations for Innovative Devices and Process Engineering," CRDF Cooperative Grants Program, Los Angeles, CA: University of Southern California, p. 10, 1996.

[6] M. Riznyk, and V. Riznyk, "Manufacturing Systems Based on Perfect Distribution Phenomenon." in Intelligent Systems in Design and Manufacturing III, vol. 4192, SPIE, Boston, U.S., 2000, pp.364-367.

[7] V. Riznyk, "Model of Intelligent Toroidal Systems of Axes," in 14th International Scientific and Technical Conference "Computer Science and information Technologies" (CSIT), Lviv, Ukraine, 2019, pp.80-83.

[8] B.Ph. van Milligen, and A. Lopez Fraguas, "Expansion of vacuum magnetic fields in toroidal harmonics," Computer Physics Communications 81, Issues 1-2, 1994, pp. 74-90.

[9] Electronic resource: https://en.wikipedia.org/wiki/Torus

[10] Manifold, https://en.wikipedia.org/wiki/Manifold

[11] Rotman, Joseph, Galois Theory (Second ed.). Springer, 1998, doi:10.1007/978-1-4612-0617-0. ISBN 0-387-985417. MR 164558

[12] J . Singer, "A theorem in finite projective geometry and some applications to number theory," Transactions of the American Mathematical Society. vol. 43, no. 3, 1938, pp.377-385.

[13] M. Jr. Hall, “Combinatorial Theory,” Blaisell Publishing Company, 1967, p. 470.

[14] V. Riznyk, "Multi-modular Optimum Coding Systems Based on Remarkable Geometric Properties of Space," in Advances in Intelligent Systems and Computing. Springer Int. Publ., vol. 512, 2017, pp. 129-148, DOI 10.1007/978-3-319-45991-2_9. 
[15] Spacetime_symmetries,

https://en.wikipedia. org/wiki/Spacetime_symmetries

[16] P. Wigner, Symmetries and reflections. Bloomington, Indiana University Press, 1970.

[17] Euclidian group, https://en. wikipedia. org/wiki/Euclidian group

[18] V. Riznyk, "Multidimensional Systems Optimization Developed from Perfect Torus Groups", International Journal of Applied Mathematics and Informatics, vol. 9, 2015, pp. 50-54.

\section{Creative Commons Attribution License 4.0 (Attribution 4.0 International, CC BY 4.0)}

This article is published under the terms of the Creative Commons Attribution License 4.0

https://creativecommons.org/licenses/by/4.0/deed.en US 